\title{
GEOMETRICAL BENCHMARKING OF LASER POWDER BED FUSION SYSTEMS BASED ON DESIGNER NEEDS
}

\author{
Montero, Joaquin (1,2); \\ Weber, Sebastian $(1,2)$; \\ Petroll, Christoph (2); \\ Brenner, Stefan (1); \\ Bleckmann, Matthias (2); \\ Paetzold, Kristin (1); \\ Nedeljkovic-Groha, Vesna (1) \\ 1: University of the Bundeswehr Munich; \\ 2: Bundeswehr Research Institute for Materials, Fuels and Lubricants (WIWeB)
}

\begin{abstract}
Commercially available metal Laser Powder Bed Fusion (L-PBF) systems are steadily evolving. Thus, design limitations narrow and the diversity of achievable geometries widens. This progress leads researchers to create innovative benchmarks to understand the new system capabilities. Thereby, designers can update their knowledge base in design for additive manufacturing (DfAM). To date, there are plenty of geometrical benchmarks that seek to develop generic test artefacts. Still, they are often complex to measure, and the information they deliver may not be relevant to some designers. This article proposes a geometrical benchmarking approach for metal L-PBF systems based on the designer needs. Furthermore, Geometric Dimensioning and Tolerancing (GD\&T) characteristics enhance the approach. A practical use-case is presented, consisting of developing, manufacturing, and measuring a meaningful and straightforward geometric test artefact. Moreover, optical measuring systems are used to create a tailored uncertainty map for benchmarking two different L-PBF systems.
\end{abstract}

Keywords: 3D printing, Additive Manufacturing, Design for Additive Manufacturing (DfAM), Laser Powder Bed Fusion, Geometrical Benchmarking

\section{Contact:}

Montero, Joaquin

University of the Bundeswehr Munich

Institute for Technical Product Development

Germany

j.montero@unibw.de

Cite this article: Montero, J., Weber, S., Petroll, C., Brenner, S., Bleckmann, M., Paetzold, K., Nedeljkovic-Groha, V. (2021) 'Geometrical Benchmarking of Laser Powder Bed Fusion Systems Based on Designer Needs', in Proceedings of the International Conference on Engineering Design (ICED21), Gothenburg, Sweden, 16-20 August 2021. DOI:10.1017/ pds. 2021.427 


\section{INTRODUCTION}

Additive Manufacturing (AM) or 3D printing is the "process of joining materials to make parts from 3D model data, usually layer upon layer, as opposed to subtractive manufacturing and formative manufacturing methodologies" (ISO, 2015). AM is considered one of the Industry 4.0 enablers (Sony, 2020) that allows designers to bring highly complex shapes to physicality, creating new design structures and enhancing the proliferation of the design freedom mindset (Bikas et al., 2019). Even though AM promises this design freedom, some manufacturability restrictions apply depending on the AM process in practice (Thompson et al., 2016). The study of these limitations and how to overcome them is known as Design for AM (DfAM). The continuous updates in AM systems make the working conditions fluctuate, resulting in very use-case specific and inoperative design heuristics. Consequently, DfAM needs to be in constant motion so designers can adjust their knowledge at the same pace.

Benchmarking is one of the means used to better understand the limitations and capabilities of an AM system. There are three benchmarking categories for AM systems: Geometrical, Mechanical and Process Benchmark (Mahesh, 2004), although within the scope of this article, only the first is tackled. To date, plenty of articles have been published on the study of geometrical benchmarking to evaluate the AM technological readiness. An extensive, yet outdated review has been done by Rebaioli and Fassi (2017), where over 60 test artefacts are summarised. Among the reasons for the intense research focus in this area are the rapid improvements of the AM systems, machine manufacturer updates and lack of industrial applications. Industrial usage is growing since AM started to be adopted as a well-established manufacturing method (Montero et al., 2019).

There has been some effort in creating generic standard test artefacts for the benchmarking of different AM processes (Yang and Anam, 2014). Since the capabilities differ significantly from system to system, it still becomes complicated to find consensus between the researchers and beat ambiguity. Generic test artefacts can give some high-level overview for each and every AM process (Rupal et al., 2018), but to fully understand the system in use, specific benchmarking artefacts have to be developed. The latter highlights an open research gap: How to overcome the design of meaningful geometrical benchmarking artefacts, accordingly to the specific working conditions and regular use that the designers are giving to a particular AM system.

A new structured approach for geometrical benchmarking of metal Laser Powder Bed Fusion (L-PBF) systems and a test artefact based on the designer's need are presented throughout this article. Although the approach is developed for a particular AM technology, it can be further expanded to other AM processes. Additional focus on the ease of measurements of the geometrical characteristics is included, as well as the targeting of meaningful DfAM recommendations. In this approach, the artefact's geometry will always depend on the application that the designers are interested to exploit in their particular AM system. Furthermore, it is applied in an exemplary study, where the design team uses two different L-PBF systems for the production of spare parts.

\section{STATE OF THE ART}

\subsection{Metal L-PBF}

Powder Bed Fusion (PBF) is one of the seven categories of AM processes defined by ISO/ASTM 52900 standards (ISO, 2015). The process uses evenly distributed powder as raw material and a focused energy source to fuse the powder along specific paths determined by sliced 3D data. The layers are produced using a build plate as a substrate. Once a layer is completed, the build plate lowers down and a new layer of powder is spread. This process is repeated until the 3D object is finished. L-PBF is a subclass of PBF processes that utilizes a laser beam as an energy source and a material spreader. Metal L-PBF uses metallic powder, which requires a building chamber that maintains an inert gas atmosphere, shielding the parts and preventing them from oxidizing during the build. Among the most popular materials for this process are the alloys AlSi10Mg, Ti6Al4V and stainless steel 316L.

A way to classify the L-PBF systems is by their build volume or build envelope. The volume is determined by the size of the substrate or platform, which can be circular or rectangular in the XY plane, and the achievable part height in Z-direction. Commercially all-rounder or medium size L-PBF systems have typically $250 \mathrm{~mm}$ x $250 \mathrm{~mm} \times 300 \mathrm{~mm}$ as build envelope. Anything below that volume is considered a small envelope system. Large envelope systems are more complex and are still in an early stage of 
development, due to the issues of maintaining a homogeneous shielding gas flow and preventing large parts from warping due to thermal deformations (Fraunhofer ILT, 2020).

L-PBF is a very complex process that involves many variables influencing the geometrical accuracy of the produced parts. For example, the powder material has a characteristic particle size distribution that depends on its production batch. The size distribution changes when the powder is re-used or mixed with some other powder, altering the print job. Mixing the powder material is a common practice for L-PBF systems to save costs. Additionally, the machine process parameters, such as laser variables and envelope temperature, determine the part's thermal cycling. These parameters are adjusted by the user or the machine manufacturer and influence the thermal distortions, which are also depending on the part's geometry.

Since there are several process variables involved, repeatability across different L-PBF systems is challenging to achieve, and results are very dependent on the working conditions. These conditions are understood as all process variables that influence the outcome of the AM process. Such variables are machine parameters, ambient conditions, raw material state and the experience of the operator.

\subsection{Benchmarking of Metal L-PBF systems}

The current fast pace in developing new L-PBF systems and software upgrades change the limitations and consequently the design constraints. This gives impulse to create new up-to-date benchmarks to enhance the existing DfAM knowledge base. Benchmarking the L-PBF technology as a whole is very complex since there are many external factors that influence the part geometry and repeatability (Dowling et al., 2020). In other words, each L-PBF system behaves in its own way, depending on the working conditions.

So far, numerous design artefacts have been released to evaluate the geometrical and dimensional accuracy of metallic L-PBF systems. Some of the most relevant are listed in Table 1. Typically, these existing works concentrate efforts in designing new artefacts with the potential to be standardised across different platforms (Yang and Anam, 2014). These works fit as many machine-challenging features as possible in one print job to expose the technological capabilities. A remarkable achievement of the latter is the recent contribution from Gruber et al. (2020), where L-PBF and other powder-based AM processes are compared using the same benchmarking artefact.

Geometrical benchmarking is not only about producing the artefact but also measuring it. The characteristic surface roughness from L-PBF interferes with dimensioning, particularly for small geometries. There are several metrological systems available to cope with this task. Yet, blue light scanners and portable hand scanners are preferred for these surfaces due to their precision, speed and performance (Giganto et al., 2020).

Table 1. L-PBF geometrical benchmarks

\begin{tabular}{|c|c|c|c|}
\hline Author & Build envelope & Artefact size $\left[\mathrm{mm}^{3}\right]$ & Material \\
\hline (Kruth et al., 2005) & Medium & $50 \times 50 \times 9$ & $\begin{array}{l}\text { Stainless steel } 316 \& \\
\text { Bronze based alloy }\end{array}$ \\
\hline $\begin{array}{l}\text { (Abdel Ghany and } \\
\text { Moustafa, 2006) }\end{array}$ & Medium & $200 \times 100 \times 40$ & $\begin{array}{l}\text { Stainless steel \& } \\
\text { Titanium }\end{array}$ \\
\hline (Moylan et al., 2012) & Medium & $77 \times 77 \times 17$ & Stainless steel \\
\hline (Kniepkamp et al., 2016) & Small (Micro) & $5 \times 5 \times 2$ & Stainless steel 316 \\
\hline (Calignano et al., 2017) & Medium & - & AlSi10Mg \\
\hline (Wang et al., 2017) & Medium & 80x80x20 (approx.) & Stainless steel 316 \\
\hline (Kamarudin et al., 2017) & Small & - & AlSi10Mg \\
\hline (Moshiri et al., 2019) & Medium & $76 \times 51 \times 18$ & Maraging Steel 1.2709 \\
\hline (Gruber et al., 2020) & Medium & $40 \times 40 \times 15$ & $\begin{array}{l}\text { Inconel } 718 \& \\
\text { Ti6Al4V }\end{array}$ \\
\hline
\end{tabular}

\section{MATERIALS AND METHODS}

Although, plenty geometrical benchmarks are available for AM systems, the vast majority of them lack a structured design procedure. Following such procedure would help users to create more meaningful test artefacts for specific applications and working conditions. Some effort has been made in this direction in the field of metal AM. The recent work of Moshiri et al. (2019) defines a benchmarking approach in 
L-PBF systems, in which four factors are constituting the experiment: Material, Design, Process and Technology. From these factors, the first three are kept locked, and the last one is used as a variable in the analysis, i.e., the only changing factor is the AM machine manufacturer. As a result, they developed an artefact that promises to give an overall characterisation of the actual level of technological readiness of L-PBF in general.

In contrast to the latter approach, this article proposes the characterization of a specific L-PBF system with a given set of regular working conditions, i.e., those working conditions that the design team faces on a daily basis. By following the ideas of the feature-based process for the design of artefacts, formulated by Rupal et al. (2018) for generic AM technologies, a process-specific approach is proposed. This approach sustains that two main objectives have to be pursued to design a new geometrical benchmarking artefact for L-PBF, as follows:

- The test artefact has to be simple. It must have a reduced number of features, to ease the measurement of geometric characteristics using standard metrology instruments, which are usually available in AM facilities. For example, any sort of 3D scanner or metrology arm.

- The measured geometric characteristics have to constitute a meaningful aid for the designers using that L-PBF system. They have to be designed for the intended applications considering the actual working conditions of the typical manufacturing scenario.

Overall, this approach seeks to support the development of test artefacts that are scalable, simpler and more specific to the application, rather than providing generic artefacts for the attempt of benchmarking a whole AM process in one print job. Additionally, the development of smaller artefacts is promoted. This encourages benchmarking even in small envelope L-PBF systems, where the majority of the available benchmarking artefacts is not suitable. The proposed benchmarking strategy can be explained as a 3-step process. The input is the user experience in that specific L-PBF system, the output a set of uncertainties for the relevant geometries. The process schematic appears in Figure 1.

In the following subsections, each step will be explained in detail using an exemplary study: A design team develops and manufactures individual spare parts in small and medium envelope L-PBF systems using recycled aluminium alloy powder. Typically, these parts are meant to replace originally casted or forged components, belonging to more extensive functional assemblies. Therefore, interconnecting features are relevant (Montero et al., 2020).

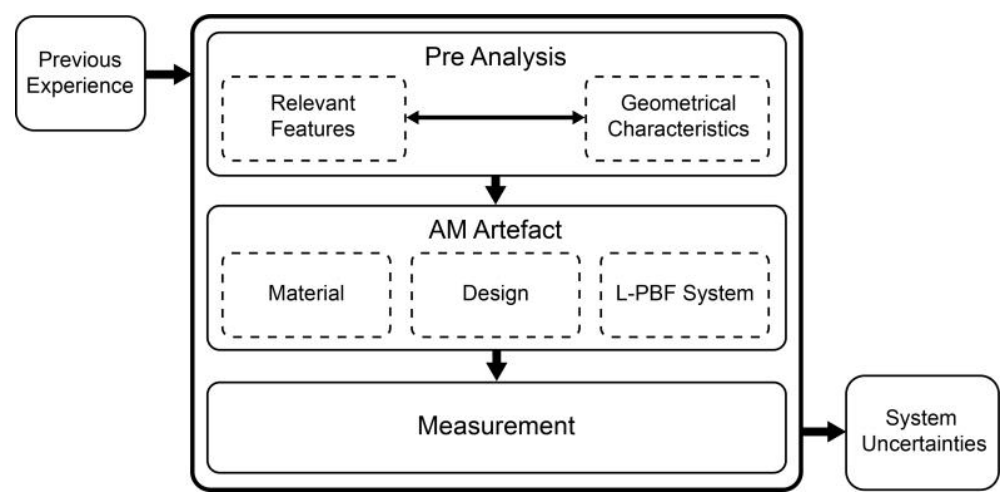

Figure 1. An approach for benchmarking an L-PBF system

\subsection{Pre-analysis}

In order to design the new artefact, designers are required to identify all relevant features that impact the part quality in their application field. This step is the start of the geometrical benchmarking process and relies heavily on the team's experience. The identification of required features leads to a set of needed geometrical characteristics (GCs), which are those geometries that define the part's physicality.

In the area of conventional manufacturing, the international standards in Geometrical Product Specifications (ISO, 2017) describe the GCs in shape, size and positional relationships with geometric tolerances. These tolerances are the allowable error. Additionally, the American standards tackle the same issue and call it Geometrical Dimensioning and Tolerancing (GD\&T) (ASME, 2018). Both systems are not fully compatible, yet the latter is the preferred standard in AM benchmarking research. Therefore, GD\&T is used throughout this article. 
Regarding AM, the study of achievable GCs is tackled by many authors, but not many of them consider or provide information on GD\&T (Rupal et al., 2018). Thus, this approach considers two groups of GCs to be included in the artefact design: The standard derived GD\&T and the AM relevant characteristics (Wang et al., 2017), which are inherent to the studied AM process. This strategy can be expanded to different AM technologies by including other GCs. Yet, for the case of the evaluation of the L-PBF process the GCs of interest are summarized in Table 2. These GCs are derived from the reviewed articles from Table 1 in combination with the mentioned standards.

Table 2. Geometric characteristics for L-PBF benchmarking

\begin{tabular}{|c|c|c|c|}
\hline \multicolumn{2}{|c|}{ GD\&T } & \multicolumn{2}{c|}{ AM relevant } \\
\hline Circularity & Cylindricity & Feature size & Wall thickness \\
\hline Perpendicularity & Angularity & True size & Surface roughness \\
\hline Parallelism & Symmetry & Repeatability & Stepping effect \\
\hline Straightness & Flatness & Overhang angle & True position \\
\hline Concentricity & Coaxiality & & \\
\hline
\end{tabular}

The design team is producing spare parts that use to be casted, therefore it is important to know the manufacturability of vertical walls, draft angles, symmetrical features and surface quality at critical building angles, as mentioned by Atzberger et al. (2018). Considering the GCs from the Table 2, the following GCs are created based on the interest of the design team. As an observation, some of the GCs are intentionally measured away from the part's base, since the largest distortions are expected at maximum part height after the separation from the build platform (Zaeh and Branner, 2010).

- GC1: The relative position of features within a $40 \mathrm{~mm}$ range at a part height of $100 \mathrm{~mm}$ to evaluate fitting and assembly considerations.

- GC 2: Symmetry in the building volume. For example, several requested parts are covers or casings, which have symmetric features as fixation points.

- GC 3: Angularity at the critical overhang angle, which is $45^{\circ}$ for most L-PBF systems (Protolabs, 2018). As an example, some spares are square or prismatic and therefore need to be placed in an inclined position relative to the baseplate. To minimize support structure, even straight features are printed in an inclined position.

- GC 4: Parallelism and perpendicularity of planes across the part height. Some casted spare parts are machined to keep orthogonality of its planes. Casting draft angles limit such surfaces to be manufactured as they should be, without the need for post-processing. Thus, GC4 is needed to figure out when such post-processing can be avoided.

- GC 5: The true position of features away from the build plate. Fixation points or links to other parts in the assembly are relevant for spare parts. Therefore, uncertainty in the critical section of the build envelope is necessary to avoid errors in the position of fixations or links.

- GC 6: True size of medium-sized features at maximum part height. To consider the distortion at the critical part section.

As it can be observed, only a few geometric characteristics from Table 2 are considered to create a meaningful and simple test artefact for this particular L-PBF system setup and conditions.

\subsection{Geometrical Benchmark Design}

The material and L-PBF system are already determined by the regular working conditions. Next, the geometrical benchmarking design has to be created in the AM Artefact step. This is performed using the features recommended in the Design for Metrology approach from Rivas Santos et al. (2020).

To evaluate GC1-3, a support-structure-free design with a base of $40 \times 40 \mathrm{~mm}^{2}$ and an inclination of $45^{\circ}$ is proposed, keeping in mind that the artefact will be manufactured in a small-envelope L-PBF system. To measure GC4, the artefact has a square shape that leads to orthogonal planes across the part height. In order to determine a relative coordinate system, an array of features at maximum part height is set. These features are used as a reference to measure the part independent from the build platform and support structure, if present. This allows the artefact to be used in L-PBF systems with a different build platform shape, e.g., circular. To create the array of features and measuring GC5-6, a cluster of $10 \mathrm{~mm}$ spheres is located on the top face at maximum artefact height. Spheres are used as a mean for determining geometrical points of interest (sphere's centres), because more precise measurements are possible and they is less dependent on the surface determination (Rivas Santos et al., 2020). 
Furthermore, the surface roughness is in the order of the layer height for L-PBF parts. Consequently, the layer stepping-effect has no influence on the determination of the sphere centre. In this way, it is possible to measure distances between these points of interest as well as geometrical uncertainties from ideal planes, which are determined by the sphere's centres.

The angularity of the down-skin at the critical angle is reported, using the relative reference system, as well as the true position of a self-supported holes array across the faces of the artefact. These holes are diamond-shaped, require no support structure and can be used as a drilling jig for further post-machining. Figure 2 shows the 3D CAD model of the designed artefact with the tags (A-D, for planes; 1-8, for spheres) for further inspection and the suggested reference coordinate system. The dimensions of the artefact are $40 \times 40 \times 100 \mathrm{~mm}^{3}$.
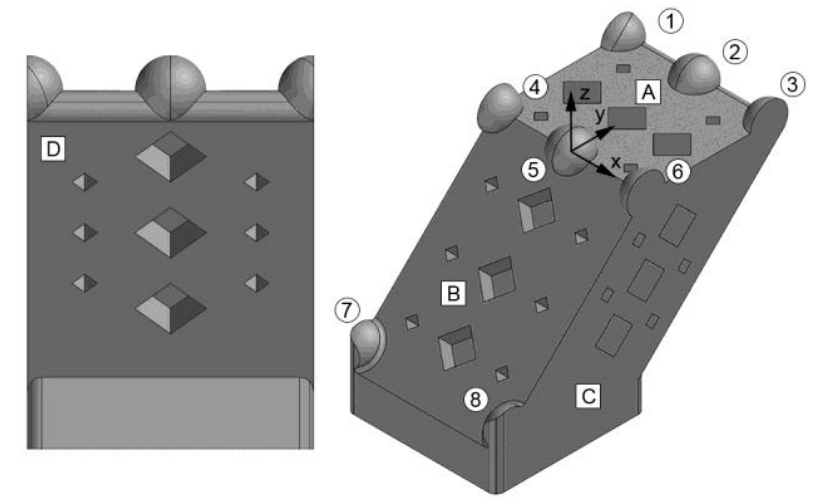

Figure 2. The benchmarking artefact $C A D$ model. planes $A-B-C-D$ and spheres 1 through 8.

\subsection{Manufacturing of the artefact}

The artefact was produced in two different L-PBF systems using recycled SLM ${ }^{\circledR}$ Solutions AlSi10Mg powder, as normally used within regular working conditions. An SLM ${ }^{\circledR}$ 125HL (referred to as System 1) and an $\mathrm{SLM}^{\circledR} 280 \mathrm{HL}$ (referred to as System 2) were used for artefact A1 and A2, respectively. Both systems have a rectangular baseplate as build-platform. System 1 has a small and System 2 a medium envelope. For both machines, the process parameters are considered to be within the standard parameters, which are recommended by the L-PBF system's manufacturer. Each machine is located in different facilities and operated by different technical staff, yet the working conditions are kept consistent over different print jobs. The condition of the artefacts is evaluated and reported in as-built condition, removed from the build-platform with no further post-processing. Figure 3 shows a picture of the manufactured artefacts.

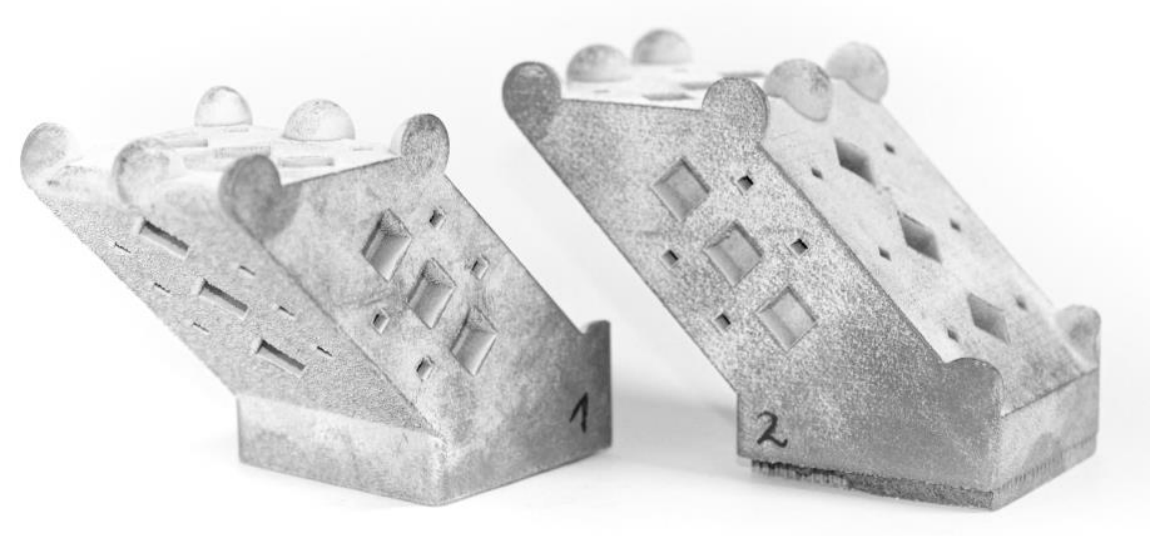

Figure 3. The artefact manufactured by different L-PBF systems

\section{EXPERIMENTAL RESULTS AND DISCUSSION}

To perform the Measurement step, optical measurement systems (OMS) are used. A 3D blue light scanner Keyence ${ }^{\circledR}$ VL-550 is utilized as the primary option, with an uncertainty of $\pm 0.01 \mathrm{~mm}$. To cross-validate the measurements and to reach the GCs that are not readable by the first OMS, a CT- 
scanner $\mathrm{GE}^{\circledR}$ v|tome|x XL300 with an uncertainty of $\pm 0.003 \mathrm{~mm}$ is used. The result of the measurements is captured as a 3D point cloud by the OMS and transformed into a high-resolution STL-file, which is a triangulated or triangular-tessellated surface. The uncertainties that this process introduces are contemplated in the reported values, although they are insignificant. Because the introduced uncertainties from both OMSs are under $0.01 \mathrm{~mm}$ for the external surfaces and in the order of the surface roughness (Majeed et al., 2019; Yang et al., 2019), it has no effect on the overall result of the measurements for creating the DfAM recommendations. Both OMSs fall into the to-go measurement methods for evaluating metallic L-PBF parts and to perform geometrical benchmarking (Giganto et al., 2020).

To execute the shape and dimension analysis, the software GOM Inspect Suite is used. The measurements of both artefacts are compared to the reference geometry, i.e., the artefact CAD model. Because of the reasons explained in section 3.2 and to highlight symmetry discrepancies, the alignment is performed using a coordinate system determined by spheres 2,5 and 6 . The latter contrasts with the common practice of using a best-fit algorithm on the external surfaces. Therefore, symmetry deviations are easier to identify. A comparison of both artefacts vs. the designed CAD model is shown in Figure 4. A view of the 3D CT-scans is shown in Figure 5.
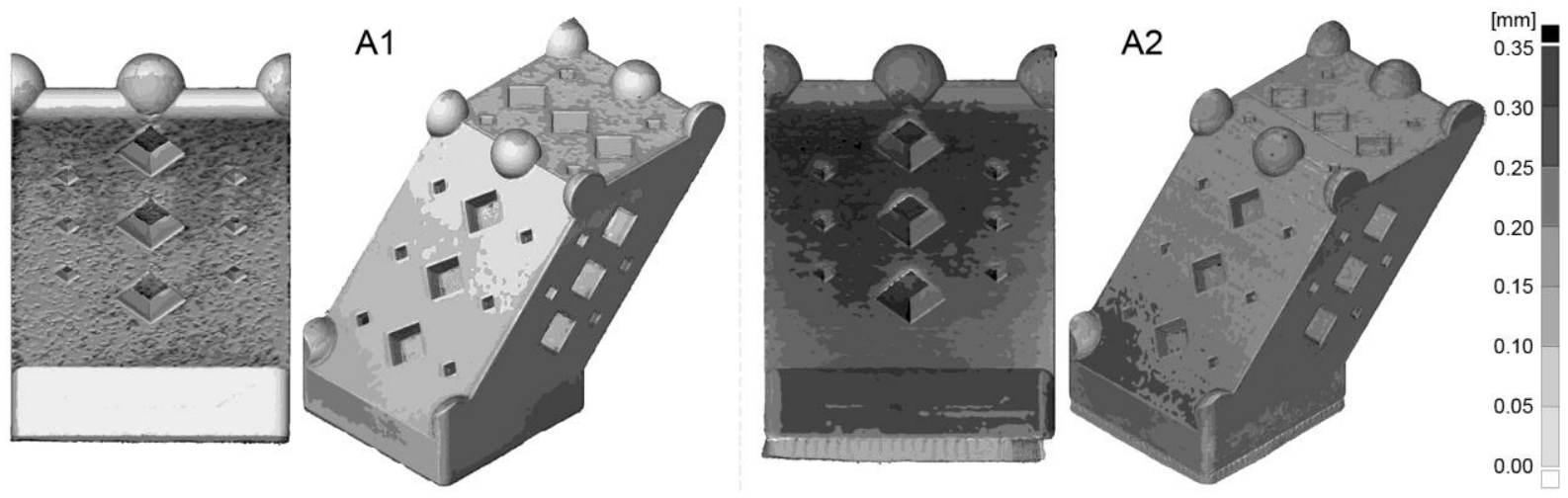

Figure 4. Geometrical comparison, 3D scan vs CAD model

A1

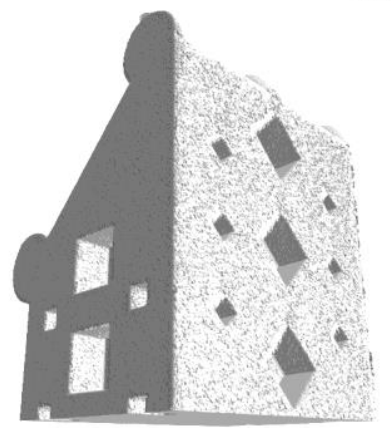

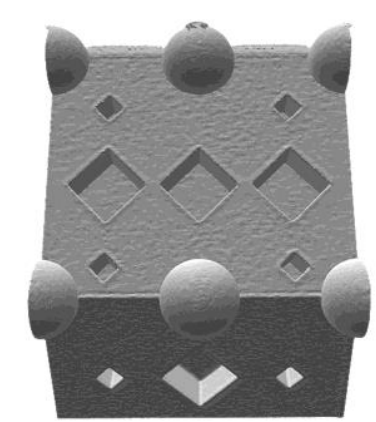

A2

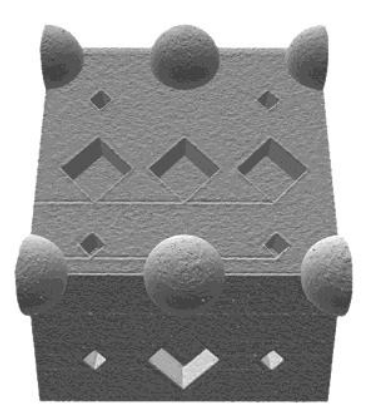

Figure 5. 3D CT-scan of the artefacts

The absolute true position and true size of the spheres were measured multiple times and compared in a table. These results are not shown in this article, as it would not provide additional value. The uncertainties are derived from the analysis of these measurements. All the uncertainties values are reported utilising the highest deviations from the design values and considering the OMSs error. The results are the geometrical uncertainties shown in Table 3, obtained by using the measuring strategy explained below. These can be used as DfAM guidelines for the analysed L-PBF systems in particular. Additionally, the CT-scans revealed no keyholes or severe porosity effects on both cases, but a considerable rougher surface on artefact 1 was observed. Particularly in the downskin (face D), as it can be observed in Figure 5. This suggests a better set of process parameter for that L-PBF system concerning the surface finish.

The measuring strategy for each GC comprehends:

$\mathrm{GC} 1$ is the true position in $\mathrm{XY}$ plane of features at the maximum part height. For this, the deviations of the outer spheres are used. GC2 is the symmetry on the building volume utilizing the centre of the 
top face A as a datum point and the plane $\mathrm{XZ}$ and $\mathrm{YZ}$. GC3 is the angularity of the face $\mathrm{D}$, using the plane determined by the centre of the spheres 2,4 and 6 as datum plane, and a design angle of $135^{\circ}$ on the $\mathrm{YZ}$ plane. GC4 is the parallelism determined using the diamond-shaped holes centres and the same datum plane as GC3, at different part heights. GC5 is the true position of features away from the build plate, determined by the highest deviation in the relative position of the centre of the hole and the spheres 7-8 from the datum plane of GC3. GC6 is the true size of medium-sized features, determined by the diameter deviation of the spheres lying in face A.

Table 3. Design uncertainties for the benchmarked geometrical characteristics in $\mathrm{mm}$.

\begin{tabular}{lcccccccc}
\hline Artefact & GC1 & GC2 $_{\mathrm{XZ}}$ & GC2 $_{\mathrm{YZ}}$ & $\mathbf{G C 3}$ & GC4 $_{30 \%}$ & GC4 $_{70} \%$ & GC5 & GC6 \\
\hline A1 & 0.08 & 0.02 & 0.12 & 0.20 & 0.04 & 0.03 & 0.03 & 0.05 \\
A2 & 0.04 & 0.03 & 0.16 & 0.03 & 0.02 & 0.02 & 0.02 & 0.42 \\
\hline
\end{tabular}

The GCs' uncertainties acquired were of great use for the design team, because they target the usual kind of parts that the team re-designs. In contrast to the more generic artefacts, the design proposed in this article provided the necessary information for these two L-PBF systems. Although both systems are considered similar, they showed discrepancies in some of the GCs. The artefacts were easy to measure and compare to the ideal geometry by using the spheres' implicit coordinate system. The squared holes were more challenging to measure, yet the CT-scan was used for such measurements delivering excellent results.

\section{CONCLUSION}

A methodology for designing geometrical benchmarks based on the designers need and given working conditions has been proposed. A practical use case led to creating a simple test artefact that allows the design team to measure meaningful uncertainties for their particular design field, using small and medium envelope LPBF systems with AlSi10Mg.

Regarding the systems evaluated in this article, an uncertainty map was created for the relevant geometric characteristics following GD\&T aspects and using a relative coordinates system. Even though A1 showed a larger surface roughness, the true positions and true sizes outperform A2. The symmetry seems to be in the same order for both systems. Therefore, it can be assumed for this case that GC2 is rather part-dependant. These results suggest that the designers have to pay more attention while parametrising a design for manufacturing in system 2 than in system 1, as long as the surface finish is not relevant. The worst deviation was observed in the GC6 for A2, where the design uncertainty reached $0.42 \mathrm{~mm}$. This highlights the fact that even similar L-PBF systems can have very different geometric performance, while manufacturing the same geometries due to the several factors involved in the process.

A clear and simple benchmarking strategy is essential to identify part- and process-related uncertainties. The main benefits of using tailored geometrical benchmark artefacts are the ease in measurement and obtainment of relevant information without further redundancies. This can aid designers to understand where to focus the design parametrisation for rapid modification in future design iterations. Furthermore, they can be used as a mean to determine system detriment over time, because users can evaluate the deviations in the features of their interest after a certain period of printing jobs. As shown, it is not feasible to develop a complete generic artefact that can benchmark all the AM systems. Also, it is neither possible to obtain one that can cover all the geometric characteristics without overcomplicating the measurements. The complete understanding of the machine's capabilities is, after all, particular for each and every use-case. Moreover, it is the system user's responsibility to know where the uncertainties can challenge the actual part design, avoid unnecessary iterations, and reach the right design parametrisation.

\section{REFERENCES}

Abdel Ghany, K. and Moustafa, S.F. (2006), "Comparison between the products of four RPM systems for metals”, Rapid Prototyping Journal, Vol. 12 No. 2, pp. 86-94. http://doi.org/10.1108/13552540610652429 ASME. (2018), Dimensioning and Tolerancing Y14.5-2018., ASME.

Atzberger, A., Montero, J., Schmidt, T.S., Bleckmann, M. and Paetzold, K. (2018), "Characteristics of a metal additive manufacturing process for the production of spare parts”, Symposium on Design for X, Vol. 29, pp. 83-94. 
Bikas, H., Lianos, A.K. and Stavropoulos, P. (2019), “A design framework for additive manufacturing”, The International Journal of Advanced Manufacturing Technology, Vol. 103 No. 9-12, pp. 3769-3783. http://doi.org/10.1007/s00170-019-03627-z

Calignano, F., Lorusso, M., Pakkanen, J., Trevisan, F., Ambrosio, E.P., Manfredi, D. and Fino, P. (2017), "Investigation of accuracy and dimensional limits of part produced in aluminum alloy by selective laser melting”, The International Journal of Advanced Manufacturing Technology, Vol. 88 No. 1-4, pp. 451458. http://doi.org/10.1007/s00170-016-8788-9

Dowling, L., Kennedy, J., O’Shaughnessy, S. and Trimble, D. (2020), “A review of critical repeatability and reproducibility issues in powder bed fusion”, Materials \& Design, Vol. 186, p. 108346. http://doi.org/10.1016/j.matdes.2019.108346

Fraunhofer ILT. (2020), "Novel LPBF Machine Concept for Additive Manufacturing of Large Components", FutureAM - Next Generation Additive Manufacturing, 21 November, p. 1. https://www.futuream.fraunhofer.de/en/news_and_media/video-lpbf-scalable-machine-concept.html

Giganto, S., Martínez-Pellitero, S., Cuesta, E., Meana, V.M. and Barreiro, J. (2020), “Analysis of Modern Optical Inspection Systems for Parts Manufactured by Selective Laser Melting”, Sensors, Vol. 20 No. 11, p. 3202. http://doi.org/10.3390/s20113202

Gruber, S., Grunert, C., Riede, M., López, E., Marquardt, A., Brueckner, F. and Leyens, C. (2020), “Comparison of dimensional accuracy and tolerances of powder bed based and nozzle based additive manufacturing processes", Journal of Laser Applications, Vol. 32 No. 3, p. 032016. http://doi.org/10.2351/7.0000115

ISO. (2015), ISO / ASTM52900-15, Terminology for Additive Manufacturing - General Principles Terminology, ASTM International. https://doi.org/10.1520/ISOASTM52900-15.

ISO. (2017), ISO 1101:2017(En) Geometrical Product Specifications (GPS) — Geometrical Tolerancing — Tolerances of Form, Orientation, Location and Run-Out, ISO, available at: https://www.iso.org/standard/66777.html

Kamarudin, K., Wahab, M.S., Raus, A.A., Ahmed, A. and Shamsudin, S. (2017), "Benchmarking of dimensional accuracy and surface roughness for AlSi10Mg part by selective laser melting (SLM)", AIP Conference Proceedings 1831.

Kniepkamp, M., Fischer, J. and Abele, E. (2016), “Dimensional Accuracy of Small Parts Manufactured by Selective Laser Melting”, presented at the Solid Freeform Fabrication 2016: Proceedings of the 26th Annual International Solid Freeform Fabrication Symposium - An Additive Manufacturing Conference, Austin, TX, USA, available at: http://utw10945.utweb.utexas.edu/sites/default/files/2016/122-Kniepkamp.pdf.

Kruth, J.-P., Van Den Broucke, B., Van Vaerenbergh, J. and Mercelis, P. (2005), "Benchmarking of different SLS/SLM processes as Rapid Manufacturing techniques", International Conference Polymers \& Moulds Innovations (PMI), presented at the International Conference Polymers \& Moulds Innovations (PMI), Gent, Belgium.

Mahesh, M. (2004), Rapid Prototyping and Manufacturing Benchmarking, PhD Thesis, National University of Singapore, Singapore, available at: http://scholarbank.nus.edu.sg/handle/10635/14697.

Majeed, A., Lv, J., Zhang, Y., Muzamil, M., Waqas, A., Shamim, K., Qureshi, M.E., et al. (2019), “An investigation into the influence of processing parameters on the surface quality of AlSi10Mg parts by SLM process", 2019 16th International Bhurban Conference on Applied Sciences and Technology (IBCAST), presented at the 2019 16th International Bhurban Conference on Applied Sciences and Technology (IBCAST - 2019), IEEE, Islamabad, Pakistan, pp. 143-147. http://doi.org/10.1109/IBCAST.2019.8667175

Montero, J., Weber, S., Bleckmann, M., Atzberger, A., Wirths, L. and Paetzold, K. (2019), "Spare part production in remote locations through Additive Manufacturing enhanced by agile development principles", 2019 IEEE International Conference on Engineering, Technology and Innovation (ICE/ITMC), presented at the 2019 IEEE International Conference on Engineering, Technology and Innovation (ICE/ITMC), IEEE, Valbonne Sophia-Antipolis, France, pp. 1-8. http://doi.org/10.1109/ICE.2019.8792631

Montero, J., Weber, S., Bleckmann, M. and Paetzold, K. (2020), "A methodology for the decentralised design and production of additive manufactured spare parts", Production \& Manufacturing Research, Taylor \& Francis, Vol. 8 No. 1, pp. 313-334. http://doi.org/10.1080/21693277.2020.1790437

Moshiri, Candeo, Carmignato, Mohanty, and Tosello. (2019), "Benchmarking of Laser Powder Bed Fusion Machines", Journal of Manufacturing and Materials Processing, Vol. 3 No. 4, p. 85. http://doi.org/10.3390/jmmp3040085

Moylan, S.P., Slotwinski, J.A., Cooke, A.L., Jurrens, K.K. and Donmez, M.A. (2012), "Proposal for a standardized test artifact for additive manufacturing machines and processes", Proceedings of the Solid Freeform Fabrication Symposium, presented at the Solid Freeform Fabrication Symposium, Austin, TX, USA, available at: https://www.nist.gov/publications/proposal-standardized-test-artifact-additivemanufacturing-machines-and-processes.

Protolabs. (2018), "How to Design and Manufacture Metal 3D-Printed Parts", Protolabs, available at: https://www.protolabs.com/resources/design-tips/how-to-design-and-manufacture-metal-3d-printed-parts/ (accessed 2 November 2020). 
Rebaioli, L. and Fassi, I. (2017), “A review on benchmark artifacts for evaluating the geometrical performance of additive manufacturing processes", The International Journal of Advanced Manufacturing Technology, Vol. 93 No. 5-8, pp. 2571-2598. http://doi.org/10.1007/s00170-017-0570-0

Rivas Santos, V.M., Thompson, A., Sims-Waterhouse, D., Maskery, I., Woolliams, P. and Leach, R. (2020), "Design and characterisation of an additive manufacturing benchmarking artefact following a design-formetrology approach", Additive Manufacturing, Vol. 32. https://doi.org/10.1016/j.addma.2019.100964.

Rupal, B.S., Ahmad, R. and Qureshi, A.J. (2018), "Feature-Based Methodology for Design of Geometric Benchmark Test Artifacts for Additive Manufacturing Processes”, Procedia CIRP, Vol. 70, pp. 84-89. https://doi.org/10.1016/j.procir.2018.02.012

Sony, M. (2020), "Pros and cons of implementing Industry 4.0 for the organizations: a review and synthesis of evidence", Production \& Manufacturing Research, Vol. 8 No. 1, pp. 244-272. http://doi.org/10.1080/21693277.2020.1781705

Thompson, M.K., Moroni, G., Vaneker, T., Fadel, G., Campbell, R.I., Gibson, I., Bernard, A., et al. (2016), "Design for Additive Manufacturing: Trends, opportunities, considerations, and constraints", CIRP Annals, Vol. 65 No. 2, pp. 737-760. http://doi.org/10.1016/j.cirp.2016.05.004

Wang, D., Wu, S., Bai, Y., Lin, H., Yang, Y. and Song, C. (2017), "Characteristics of typical geometrical features shaped by selective laser melting”, Journal of Laser Applications, Vol. 29 No. 2, p. 022007. http://doi.org/10.2351/1.4980164

Yang, L. and Anam, M.A. (2014), "An investigation of standard test part design for additive manufacturing", Proceeding of the Solid Free Form Fabrication Symposium, pp. 901-922.

Yang, T., Liu, T., Liao, W., MacDonald, E., Wei, H., Chen, X. and Jiang, L. (2019), "The influence of process parameters on vertical surface roughness of the AlSi10Mg parts fabricated by selective laser melting", Journal of Materials Processing Technology, Vol. 266, pp. 26-36. http://doi.org/10.1016/j.jmatprotec.2018.10.015

Zaeh, M.F. and Branner, G. (2010), "Investigations on residual stresses and deformations in selective laser melting”, Production Engineering, Vol. 4 No. 1, pp. 35-45. http://doi.org/10.1007/s11740-009-0192-y 